\title{
A Survey of Modern Approach for Cloud Service Recommendation based on Security
}

\author{
Shital Sumbe \\ Department of computer \\ Engineering Jspm's Jscoe, \\ Pune, Maharashtra, India
}

\author{
Sujata Jadhav \\ Department of computer \\ Engineering Jspm's, Jscoe, \\ Pune, Maharashtra, India
}

\author{
M.V. Pawar \\ Department of computer \\ Engineering Jspm's Jscoe, \\ Pune, Maharashtra, India
}

\begin{abstract}
CC (Cloud computing) is a new generation of utility computing and highly growing phenomenon in present IT industry. As the number of new cloud service provider increases, user require secure, reliable and trustworthy Cloud Service Provider from the market to store confidential data. $\mathrm{CC}$ provide the architecture for creating clouds with marketoriented recourse allocation by leveraging technologies such as virtual machine (VMs). VCC(Vehicular cloud computing) is a new technology which can be considered as hybrid technology, which has the remarkable impact on road by instantly using vehicular resources like computing, storage and internet for decision making and also remarkable impact on traffic management. The first discourse is on survey for an easy but comprehensive definition of $\mathrm{CC}$ and understanding its main aspect and gaps. An idea of trusted third party application called as Auditor, which is additional application in between the customer that is client and cloud service provides which is nothing but servers.
\end{abstract}

\section{General Terms}

Cloud service providers can be ranked based on different security measures like Digital signature, Directory attack, DDos attack, DOS attack. For this attack detection different types of algorithm used like SHA algorithm, hashing algorithm.

\section{Keywords}

Cloud computing, Cloud service provider, Trusted third party approach, Vehicular cloud computing, Mobile cloud computing, Inter Cloud

\section{INTRODUCTION}

Cloud computing is new technology used in now a days and cloud computing has emerged as a paradigm to deliver ondemand resource (infrastructure, software, platform) to its customer. cloud computing provide the architecture for creating clouds with market-oriented resource allocation by using technologies such as virtual machine(VMs) and this architecture also provide insights on market-based resource management strategies that encompass both customer driven service management and computation risk management to sustain service level agreement (SLA) oriented resource allocation

Cloud computing is based on trust and trust is crucial factor that it affects the appetite of an entity to consume a particular service or product offered by other entity. This example can be seen in everyday life where trust decisions are made. When purchasing a specific product, concentration is on certain brands due to trust that these brands will provide excellent quality compare to the unknown brands.
Service level agreements between greed user and provider have been design as mechanism for determining user's quality of service for this purpose requirements are gathered. Provider is able to determining utility from its environment. Bilateral protocol for SLA negotiation is used for negotiation between resource broker and provider for additional reservation to compute node and evaluate it on real grid system. Cloud computing gives right to customer for dynamically scaling their application software platform, hardware infrastructure according to predefined Service level agreements.

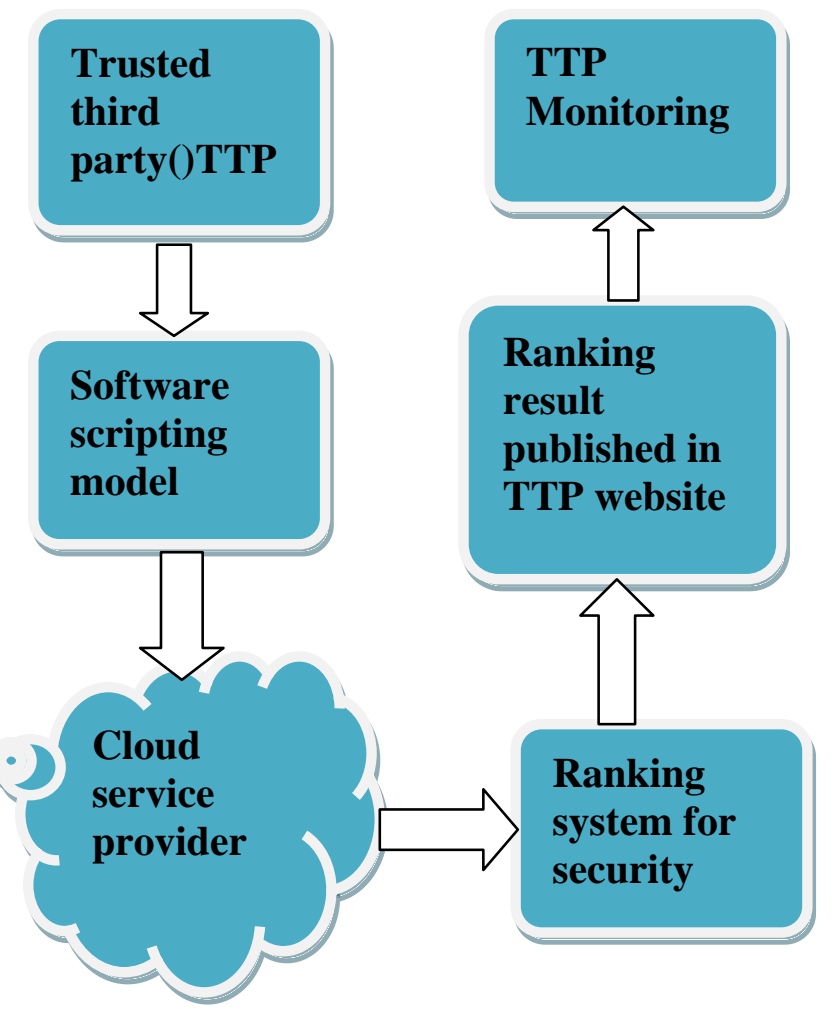

Fig 1: Conceptual model for CSP recommendation.

Resources available in single cloud data centre are limited if there is demand of an elastic application is recognized in a given time then cloud provider will not be able to give uniform quality of service to handle such demand and SLA get violated. So because of this inter cloud and cloud coordinator scenario is proposed because increase of in public cloud provider or customer cloud provider.

Cloud consumer face different challenges such as the security, privacy and discovery of reliable resources providers. Cloud computing gives right to customer for dynamically scaling their application software platform, hardware infrastructure 
according to predefined Service level agreements. Resources available in single cloud data centre are limited if there is demand of an elastic application is recognized in a given time then cloud provider will not be able to give uniform quality of service to handle such demand and SLA get violated. So because of this inter cloud and cloud coordinator scenario is proposed.

Cloud computing is the technology for next generation information and software enabled work that is capable of changing the software working environment. With the single cloud service provider there might be the risk of service availability failure and inside theft of data. Cloud computing is not a completely new concept. It has connection to the established grid computing paradigm and related technologies such as utility computing.

Cloud computing can be viewed as the transformation into reality of a long held dream called "computing utility". To make it more marketable for user community one needs to address a variety of information security risks. Companies do not even need to plan for their growth in advance with this new "pay as you go" system. Its great potential for utility, scalability and instant access features.

$\mathrm{VCC}$ (Vehicular cloud computing) is a new technology which can be considered as hybrid technology, which has the remarkable impact on road by instantly using vehicular resources like computing, storage \& internet for decision making \& also remarkable impact on traffic management. However there is an improvement in software, hardware \& communication technologies that are empowering design \& implementation of the different types of network in different environment.

\section{RELATED WORK}

Cloud computing is new technology used in now a days and cloud computing has emerged as a paradigm to deliver ondemand resource (infrastructure, software, platform) To it customer. Cloud computing provide the architecture for creating clouds with market-oriented resource allocation by using technologies such as virtual machine (VMs). The cloud computing is based on trust and trust is crucial that it affects the appetite of an entity to consume a particular service or product offered by other entity. Bilateral protocol for SLA negotiation is used for negotiation between resource brokers and provide for additional reservation to compute node and evaluate it on real grid system. With the single cloud service provider there might be the risk of service availability failure possibility and inside theft of data.

\section{LITERATURE REVIEW}

\subsection{Existing System}

(2007) In IT cloud environment consumers rely on cloud providers to supply more of their cloud computing needs they will require specific QoS(Quality of service) to be maintained by their providers in order to meet their objective and sustain their operation. Cloud providers will need to consider and meet different QoS parameters of each and every consumer as negotiated in specific SLAs. To achieve this, cloud provider can no longer continue to deploy traditional system-centric resource management architecture that do not provide incentives for them to share their resources and still regard all service requests to be of equal importance.

(2008)SLA motivate for research \& development of formulating negotiating and establishing such agreement between users and provider. Grid system also integrate SLA specification exchange \& determining capabilities for task scheduling, Resource brokering \& resource provisioning. The open grid forum is used for creation \& specification of SLA. This is called WS-agreement. WS-agreement uses contract net protocol for exchange of SLA between user and provider.

(2010)Novel models of reputation and trust have been developed in e-market place to provide reliable services of security since traditional solution to security issues do not adequately protect providers and services consumers. The most important aspect of these models is the information relating to past behavior of customer. This information is used to present the reputation of these users in terms of availability, reliability, and security. As a centralized architecture of online reputation model, E-bay and Amazon exemplify this method. Their systems are implemented based on a centralized rating model so that users and sellers can rate each other using numerical ratings or feedback comments. Users can obtain a reputation profile for a given user to decide whether or not to proceed with a transaction with this user. For example, E-bay uses $1,0,-1$ scales which means positive, neutral, and negative respectively. The existing works in trust and reputation management system from literature. The main objectives are to provide fundamental knowledge on trust for digital environments. The current state of art in trust and reputation management systems is presented. To date, there are many trust solutions have been proposed for each environment (i.e. peer-to-peer, multi-agent system, ecommerce,).Trust in a specific context is also time-dependent which represents another important element of trust. The time-dependent of trust into three categories: time spot, time slot, and time space. Time spot is a specific time when the interaction between the trusting entity and a trusted entity occurs and a trust value is assigned. Time slot is defined as a period of duration between two time spots in which the trust values are collected. Time space is defined as the total duration of interaction between the trusting entity and a trusted entity in which all trust values of different time-slots are aggregated.

(2010)The system is already available does not provide support for load available does not support for load distribution among several cloud based data centers that determine optimal location. This optimal location is used for hosting application service to adopt reasonable Quality of service levels because of this cloud service provider are unable to determine geographic distribution of services that are consumed by user. Hence the load coordination must done automatically \& distribution of service must change in response to changes in load.

(2012)Propose system is used for investigating how multicloud deployment can reduce security risk by using security algorithm. In this work is survey the recent research on single cloud and multi-cloud to address the security risk and solution. The services can be used by cloud users in the pay per use fashion without investment .Cloud service provider such as IBM, Microsoft, Amazon and Oracle and so on are provide various kind cloud services. We found that much research has been done to ensure the security of single cloud storage whirs multi-cloud have receive less attention in the area of security

(2012)Resources available in single cloud data centre are limited if there is demand of an elastic application is recognized in a given time then cloud provider will not be able to give uniform quality of service to handle such demand and SLA get violated. So because of this inter cloud and cloud 
coordinator scenario is proposed because increase of in public cloud provider or customer cloud provider.

(2012)The purpose of this paper icloud provider should address privacy and security issues as a matter of high and urgent priority. Delaying with single cloud provider becoming less popular with customers due to potential problem such as service availability failure and the possibility that their malicious insider in the single cloud.

(2012)Now here cloud computing mainly focus on gaps and security concerns. Based on that identification of top security threats and investigates the challenges in implementing threat remediation. Then the main focus is on gapes and their proposed solution is presented. A variety of model performances measurement tools are applied to verify the model attack predilection capability. The observation made on support vector machine technique from statistical machine learning theory is able to identify the top attacks. We have to detect the activities using performances data from the hypervisor and its guest operating system which is generated by cloud customers using built in software.

(2013)This architecture discuss several interrupting application scenarios. Security \& privacy issues key management strategies' \& formation of VCC Also discussed VCC security challenges as authentication, security vehicular communication, location \& localization security. It uses a 'cryptographic algorithm' to protect vehicular communication against wide ranges of attacks and EIGamal signature scheme is the way in $\mathrm{VC}$ environmental to create a secure communication.

(2013)Here the observation made is taken into consideration that the CSP side security issues and ability to adopt of security strength by introducing Trusted Third Party(TTP) approach which provide us CSP(Cloud service provider) ranking system. To the best of knowledge, using different types of attack to protect and recognize customer interest and confidence by using security ranking systems to select secure and best CSP is the first time in MCC (Mobile cloud computing).First, TTP uses automated software scripting for checking security issues in CSP side by using software scripting to break the security strength and ability of the CSP. Therefore, considering issues regarding to security that gives customer satisfaction, previous down time, location. factors, TTP gives a secured CSP ranked system in their website. The deployment of TTPs is divided by region wise and then again extended globally, which can be called as federated TTP cloud monitoring system. Furthermore, proposed model provides confidence and trust about the new cloud customer to select the best cloud service provider according to security and trust thus proposed Conceptual Model of Federated CSP Ranking System (CMFCSPRS) gives additional security in the MCC area.

\subsection{Existing Research Gap}

[3]The architecture for market-oriented allocation of resources within clouds and also presented a vision for the creation of global cloud exchange for trading services. In this some representative platform for cloud computing covering the state-of-art .In particular, cloud IT platform presented various cloud efforts in practice from the market-oriented perspective to reveal its emerging potential for the creating third-party services to enable the successful adoption of cloud computing, such as meta negotiation infrastructure for global cloud changes and provide high performance context delivery via 'storage cloud'.
[12]Here negotiation mechanism for advance resource reservation is proposed. For this purpose negotiation protocol is used for service level agreement. Negotiation protocol allows party to make changes in proposal .This negotiation mechanism is implemented to enable user who can hold the resources for reserving nodes on shared computing resources. Negotiation protocol is bilateral protocol that consist of proposer and responder. Proposer initiate the protocol and responder replies to the protocol. Gradius broker is used to implement economy based scheduling of grid resources. Advance reservation system gives guarantee for meeting user's quality of service requirement for execution of dead line and budget.

[4]The SLA agent or model is involved in three main tasks in the proposed architecture. The first task is based on the SLA management which should effectively divide the consumer classes into different groups. The IT business objectives of consumers are used to select different types of SLA agreements

From among existing templates of SLA. The management unit of the SLA then starts the negotiation with the cloud consumer and finally, the contract must be signed. The second task for the SLA agent is business activities monitoring. The monitoring and auditing of business rules and business activities are essential to assign responsibility in the case of violations. Development is done using this three task because SLA metrics templates are designed to use business parameters such as consumers' profit. So, cloud providers should agree to pay some fees when the consumers' profit which is related with as selected service decreases.

[7]Proposed architecture mainly focus on functionalities of digital environments are to allow entities to share their resources, information, knowledge, services. and also to enable transactions between entities in open, heterogeneous and dynamic environments. Whether the successful of digital environments in achieving these functionalities can only be realized if its member entities are able to trust each other. To ensure trust relationship is nurtured, there must be a framework to determine, measure, and compute the trustworthiness value of any entity in the environment. Further, this framework must also encourage entities involvement to provide their recommendations to others. Developing an effective trust framework has proven to be difficult. This is due to the multi-faceted concept of trust that encompasses the four main elements: belief, reputation, context, and time. Furthermore, the requirement of highly performing digital environments that encourage decentralized approach gives additional challenge in developing an effective trust framework.

[8]Inter cloud environment that provide environment that is based on just in time, opportunities and scalable application service. The application services continuously and and achieving Qos targets under variable workload resource and network condition. The aim is to design an environment that support dynamic expansion of capabilities like VMs ,services, storage and database for handling sudden variation in service Cloud computing gives right to customer for dynamically scaling their application ,software platform ,hardware infrastructure according to predefined Service level agreements.

[9]Resources available in single cloud data centre are limited if there is demand of an elastic application is recognized in a given time then cloud provider will not be able to give uniform quality of service to handle such demand and SLA 
get violated. One approach is designed to avoid such scenario is enabling further following of application by scaling it across multiple independent cloud data center. This approach is involved in inter cloud project is realized by cloud coordinator. Cloud co-ordinater are agents. It allows to increase a performance, reliability, scalability of elastic applications. Here both an architecture for such such cloud coordinator and an extensible environment that allows adoption in different public and private clouds. An evaluation of cloud coordinator prototype running in small scale approach that shows the effectiveness of proposed system and its effect on elastic application.

Security for multi-cloud using a RC5 algorithm. RC5 algorithm provide security for data which are stored in multicloud instated of single cloud.RC5 algorithm used for encryption, multi-cloud computing can be applied to the data transmission security. Transmission of data will be encrypted multi-cloud computing can be applied to the data transmission restored .Only user knows the key the multi-cloud do not know the key of that transmission. In addition the loss of service availability has caused the many problems foe a large number recently data intrusions leads to many problems for the user of cloud computing.

[11]However there are some areas which are still remain unexplored for researches which includes physical location of attacker inside the same server, security \& privacy aware data sharing, data indexing, high mobility. Therefore it requires new researches on this so that VCC can be the next technology shifting paradigm that provides solutions which are feasible \& economical by converging intelligent vehicular network towards traffic management, vehicular control perception system.

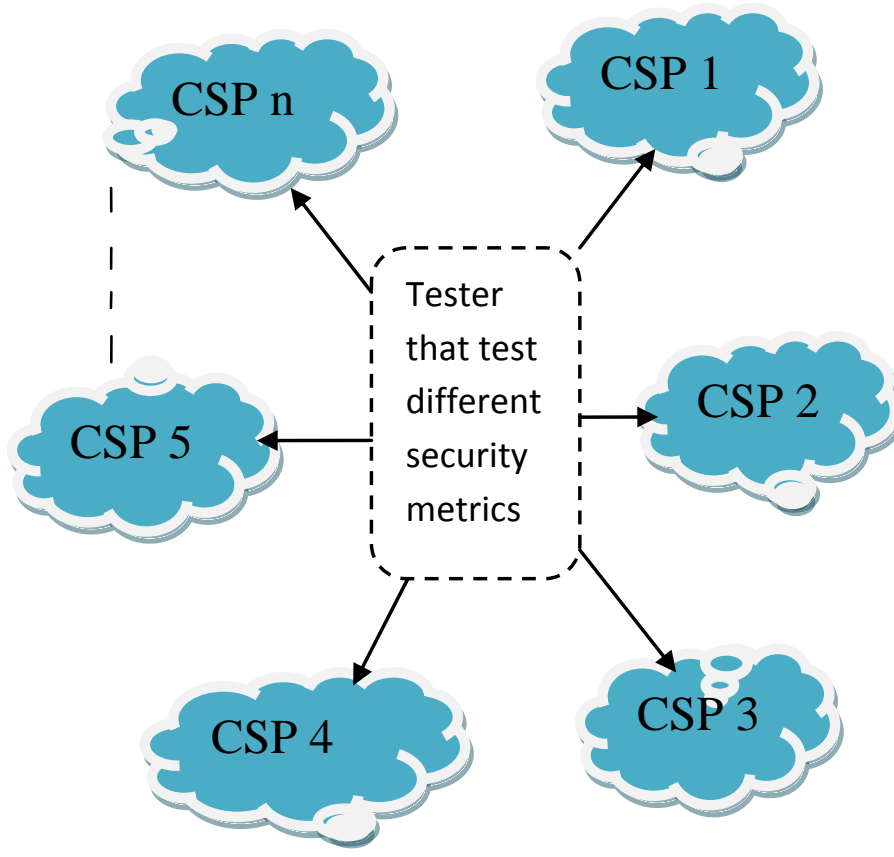

Tester:

1.Test data integrity. 4.Test dictionary attack.

2. Test dos time.

3.Generate one time password.

Fig 2: Conceptual model CSP ranking based on security measures.
[1]The model that is already proposed suggest cloud service provider according to security and trust of customer. But the model that focus on security metrics like one time password, dictionary attack, denial of service attack, data integrity will be proposed. The conceptual model is shown in Fig 2. This conceptual model consist of tester. Tester test available cloud service provider for security issues like dos time one time password, dictionary attack, data integrity. Among all available CSP, the CSP that gives best performance according to all security metrics can be indentified. Model that is shown in Fig 2 can be used for ranking cloud service provider based on security measures like reliability, performance, data integrity denial of service attack, dictionary attack, one time password. Among all cloud service provider the CSP which gives best performance according to the security metrics mentioned above. Recommendation of that CSP will be given to the customer who want to buy secure CSP.

\section{ALGORITHMS USED}

New online algorithms are used for energy aware placement and live migration of virtual machine between clouds. Here multiple heterogeneous optimization algorithm is used. It is used for market and policy engine. Here the load balancer allows round robin algorithm and least connect algorithm for routing application service request.RC5 algorithms used for encryption, multi-cloud computing can be applied to the data transmission security. Transmission of data will be encrypted multi-cloud computing can be applied to the data transmission restored .Only user knows the key .the multi-cloud do not know the key of that transmission. Digital signature algorithm like SHA1, Hash function can be used. Then for extending the security of cloud service provider, security metrics like cryptography, dos time, MD5 algorithm are taken into consideration. So by implementing this type of model recommendation of best CSP will be given to cloud customer

\section{CONCLUSION}

Federated cloud computing model offers significant performance gain for increasing response time and because of this cost is also reduced under dynamic workload. Performance of System is reliable, Scalable. Enable 'choice' the first discourse is on survey for an easy but comprehensive definition of cloud computing and understanding its main aspects and gaps. The second discourse is on thoughts for some novel approach to identify cyber-attack type using the modern machine learning techniques. Identification of CSP side security issues and tolerance of security strength is done, that provide CSP ranking system. To the best of knowledge, using attack vectors to protect and ensure customer interest and confidence by issuing security ranking systems to select secure CSP is the first time in Cloud Computing. Therefore, considering several non-measurable metrics such as customer satisfaction, Security, availability factors, CSP ranking will be done.

\section{ACKNOWLEDGMENT}

This work is supported by JSPM's Jayawantrao Sawant College of Engineering, Pune Maharashtra. First and foremost, we would like to thank our guide Prof. M.V. Pawar. Providing us with their invaluable support, motivation, suggestion and guidance throughout the course of the paper. We would like to express our gratitude towards Prof. A. S. Devare whose support and consideration has been a valuable asset during course of this paper. We convey our gratitude to our respected HEAD OF DEPARTMENT, Prof. H. A. Hingoliwala for his motivations and guidance throughout the 
work. And, last but not least we would like to thank Principal Dr. M. G. Jadhav for directly and indirectly help us for this work

\section{REFERENCES}

[1] Md Whaiduzzaman, Abdullah Gani "Measuring Security for Cloud Service Provider: A Third Party Approach." International Conference on Electrical Information and Communication technology(EICT),2013

[2] S. Venugopal, X. Chu, and R. Buyya, "A Negotiation Mechanism for Advance Resource Reservation using the Alternate Offers Protocol", Proceedings of the 16th International Workshop on Quality of Service, IWQoS'08

[3] R. Buyya, C. S. Yeo, S. Venugopal, J. Broberg, and Ibrandic, "Cloud computing and emerging IT platforms. Vision, hype and reality for delivering computing as the 5thutility,"Future Generation Computer Systems, vol.25, pp.599-616,6// 2009

[4] M. Alhamand, T. Dillon and E. Chang." SLA- Based Trust Model for cloud computing," in Network-Based information Systems (NBiS), 2010 13th International conferences on 2010, pp. 321-324.

[5] IEEE Computer Society, Enschede, Netherlands, 2008, pp. 40-49.Zibinzheng, Yeleizhang and Michael R.lyu, "Cloud Rank: A QoS driven component framework ranking for cloud computing", 2010 29th IEEE International Symposium on Reliable Distributed System.

[6] Zibinzheng, Yeleizhang and Michael R.lyu, "Cloud Rank: A QoS driven component framework ranking for cloud computing"2010 29th IEEE International Symposium on Reliable Distributed System.

[7] Geoff Skinner, Rukshan Athauda " A Holistic Review on Trust and Reputation Management System fir Digital
Environments" in International journal of computer and information Techology ( 2277- 0764),september2012.

[8] M. Whaiduzzaman M. Sookhak, A. Gani, and Buyya "A survey on vehicular cloud computing." Journal of Network and Computer Applications August 2013.

[9] S. K. Garg, S. Versteeg, and R. Buyya, "A framework for ranking of cloud computing services," Future Generation Computer Systems, vol. 29, pp. 1012-1023, 6// 2013.

[10] Abolfazli S, Sanaei Z, Ahmed E, Gani A, Buyya R "Cloud-based augmentation for mobile devices: motivation, taxonomies, and open challenges. In: IEEE Communication Surveys and Tutorials," June 2013.

[11] M. T. Khorshed, A. B. M. S. Ali, and S. A. Wasimi, "A survey on gaps, threat remediation challenges and some thoughts for proactive attack detection in cloud computing," Future Generation Computer System, vol. 28, pp. 833-851, 6// 2012.

[12] R. Buyya, C.S. Yeo, S. Venugopal, "Market-oriented cloud computing: vision, hype, and reality for delivering it services as computing utilities," 2008.

[13] R. Buyya, R. Ranjan and R. N. Calheiros, "Inter Cloud: Utility-Oriented Federation of Cloud Computing Environments for Scaling of Application Services", in : Proceedings of the 10th International Conference on Algorithms and Architectures for Parallel Processing, ICA3PP'10, Springer, Busan, South Korea, 2010, pp. $13-31$.

[14] Rodrigo N. Calheiros, Adel Nadjaran Toosi, Christian Vecchiola and Rajkumar Buyya, "A coordinator for scaling elastic applications across multiple clouds", Future Generation Computer Systems, Volume 28, No. 8,Pages: 1350-1362, ISSN: 0167-739X, Elsevier Science, Amsterdam, The Netherlands, October 2012 